\title{
Exploring selective neural electrical stimulation for upper limb function restoration
}

\author{
Wafa Tigra (1,2), David Guiraud (1), David Andreu (1,3), Bertrand Coulet (4), Anthony Gelis \\ (6), Charles Fattal (5), Pawel Maciejasz (7), Chloé Picq (7), Olivier Rossel (1), Jacques \\ Teissier (8), Christine Azevedo Coste (1)
}

(1) INRIA, LIRMM, Montpellier, France; (2) MXM, Sophia-Antipolis, France; (3) Université de Montpellier, Montpellier, France; (4) CHU Lapeyronie, Montpellier, France; (5) CRF Divio Dijon, France; (6) Propara Rehab. Center, Montpellier, France; (7) AXONIC, SophiaAntipolis, France; (8) Clinique Beau Soleil, Montpellier, France

This article is distributed under the terms of the Creative Commons Attribution Noncommercial License (CC BY-NC 4.0) which permits any noncommercial use, distribution, and reproduction in any medium, provided the original author(s) and source are credited.

\begin{abstract}
This article introduces a new approach of selective neural electrical stimulation of the upper limb nerves. Median and radial nerves of individuals with tetraplegia are stimulated via a multipolar cuff electrode to elicit movements of wrist and hand in acute conditions during a surgical intervention. Various configurations corresponding to various combinations of a 12poles cuff electrode contacts are tested. Video recording and electromyographic (EMG) signals recorded via sterile surface electrodes are used to evaluate the selectivity of each stimulation configuration in terms of activated muscles. In this abstract we introduce the protocol and preliminary results will be presented during the conference.
\end{abstract}

Key Words: electrical stimulation, neural selectivity, grasping function restoration

Eur J Transl Myol 201626 (2) 161-164

Prevalence of complete spinal cord injury (SCI), is estimated at 2 million people worldwide. SCI consequences are often devastating for patients. For trauma at cervical levels (tetraplegia), among many dysfunctions, one of the most impressive is the loss of use of four limbs.

In complete SCI, sub-lesional peripheral nervous system (PNS), does not receive anymore commands from the central nervous system (CNS) because communication is impeded. Although some assistive devices allow them to palliate basic functions, recovery of grasp movements is still seen as a priority for these patients to regain autonomy ${ }^{1,2,3,4}$. To restore hand movements, electrical stimulation remains almost the only solution. Indeed, applying an electrical current sufficient to excite cells (neurons or myocytes) allows the initiation of action potentials responsible for muscle contraction. Moving paralyzed limbs after such trauma becomes possible ${ }^{5,6,7,8,9}$. Implanted systems, such as the Freehand System (Neurocontrol, USA) or the FESMate (Japan) use epimysial or intramuscular electrodes to activate directly muscles to restore movements of the upper limb, while in the non-implanted systems, such as the Bionic Glove (Canada) or the Handmaster (Israel), the stimulation is delivered through surface electrodes. In implanted systems, activation of each muscle requires the use of at least one electrode, complexity of the device and number of foreign bodies may be high, up to 12 channels for the Freehand for instance. Risks of failure, externalization of foreign bodies and infections spreading along wires are further increased. Moreover, the needed surgery involves multiple procedures and takes considerable time and care to be successfully achieved. In one study ${ }^{10}$, it has been necessary to reoperate on four subjects over nine to make adjustments of the system while three other surgeries have been required to replace or remove broken electrodes or exchange an implant/receiver. However, patients become more independent of daily living activities, thus limiting the needs of a human aid. Although these devices have emerged as one of the most promising techniques for the restoration of hand function for SCI or stroke subjects, their use is still very limited in terms of acceptability, efficacy and trade-off between benefit / risk. Indeed, the higher the patient's lesional level is, the larger the number of muscles to be stimulated to achieve gripping movements is. A method which allows to activate more than one muscle by electrode becomes relevant. Another approach has been used for decades; functional surgery, which is mainly based on muscle-tendon transfers and opened a wide field of improvement of the functional potential of 


\section{Exploring selective neural electrical stimulation for upper limb function restoration}

Eur J Transl Myol 26 (2) 161-164

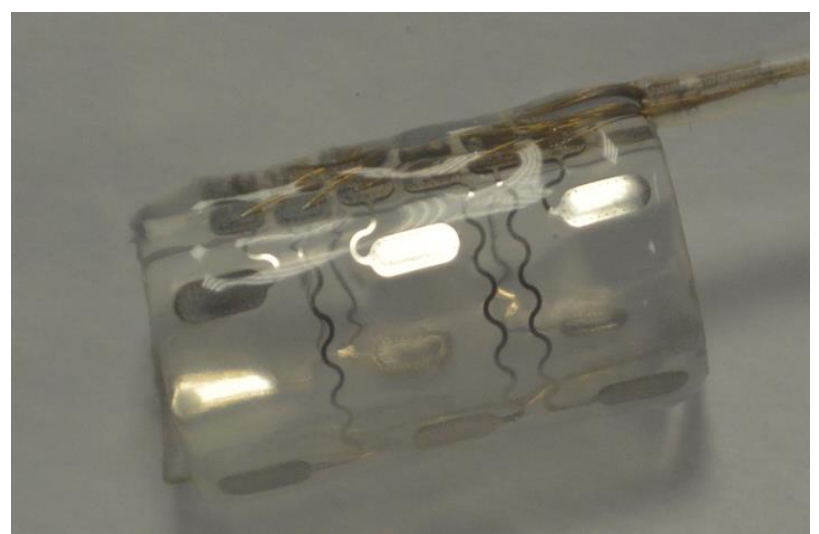

Fig 1. 12-poles multipolar cuff electrode

tetraplegics ${ }^{11,12}$. In these procedures, the distal portion of a functional tendon-muscle is detached from its natural insertion point and then fixed on a non-functional adjacent tendon in order to give back original function of the non functional tendon. For example, transfer of the biceps brachii muscle on triceps brachii muscle may allow restoring an active elbow extension, residual elbow flexion being provided by the other flexors (brachialis and brachioradialis). However, this type of approach requires the presence of a sufficient number of muscles under voluntary control, which is not always possible. Moreover, the post surgical rehabilitation does not systematically allow the recovery of the desired movement ${ }^{13}$. Keith et al. ${ }^{7}$ combined epimysial FES and muscle-tendon transfert in the Freehand implantation but their procedure was based on epimysial electrodes that needs to spread the system over all the used muscles. Therefore, if we combine muscle-tendon transfer and selective neural FES, patients with no forearm voluntary movements but with biceps and deltoid muscles still under voluntary control, could recover an active elbow extension via tendon transfer and hand movements via neural FES. Thus, the combination of a tendon transfer surgery and a multipolar electric neural stimulation (number of poles $\geq 4$ ) would optimize muscle residual activity while making the electrostimulation device less cumbersome to implement. Indeed, placed above nerve junctions, multipolar electrodes would allow selective activation of several fascicles of the same nerve. This selective activation could potentially activate different hand functions and / or muscles. Control of multiple functions via a single electrode could reduce the number of foreign bodies and electrodes. Besides, the needed energy to activate muscle decreases compared to epimysial or intramuscular stimulation. Indeed, a neural stimulation requires 10 times less energy. In the following, we present our ongoing research protocol. In acute conditions during a surgical intervention, we stimulate upper limb nerves of tetraplegic patients using multipolar cuff electrodes, to selectively activate movements of wrist and hand.

\section{Materials and Methods}

\section{Subjects}

Subjects are spinal cord injured with lesional level up to C7, AIS A or B with positive electrical mapping with a minimum score of $4 / 5$ on the Medical Research Council (MRC) Scale for at least one extensor among Extensor Carpi Radialis Longus (ECRL), Extensor Carpi Radialis Brevis (ECRB), Extensor Digitorum Communis (EDC), Extensor Pollicis (EPL) Longus or one flexor among Flexor Pollicis Longus (FPL), Flexor Digitorum Superficialis (FDS), Flexor Digitorum Profundus (FDP). Age is between 18 and 65 years and patients are in a stable neurological state for at least 6 months.

An information note explaining the aim of the study is delivered to the participants who sign an informed consent. The study is conducted, during musculotendinous transfers of arm or forearm muscles, at the Private Hospital Beau Soleil and the University Hospital Center Lapeyronie, Montpellier, France. Ethical agreement was obtained (CPP Sud Mediterrannée IV, Montpellier, France, February 10th, 2015).

\section{Multipolar electrical stimulation}

\section{Cuff electrodes}

Multipolar cuff electrodes (length 20mm, 12 oblong contacts of $2,2 \mathrm{~mm}^{2}$, designed by CorTec $\mathrm{GmbH}$, Freiburg, Germany (Fig.1)) are placed around the median and/or radial nerves next to the elbow joint. The 12 contacts are positioned to have 3 rings of 4 contacts positioned at $90^{\circ}$ from each other. The 12 contacts can be independently activated via a dedicated software and stimulator. Electrodes were designed to be positioned optimally on the median and radial human nerves.

\section{Electrical stimulator}

The neurostimulator, $R \& S T I M 12$, (designed jointly by MXM-AXONIC company and INRIA-DEMAR lab) allows the control of the 12 individual poles and 1 reference. All poles are addressed simultaneously to generate $3 \mathrm{D}$ current spreading over the 12 contacts. A synchronization output allows interfacing with signal recorders. Each active pole (i.e. implied in the electrode configuration) can be defined as a cathode or an anode. R\&STIM 12 is operated from a dedicated PC software (Lunatum developed by MXM-AXONIC company) which allows to program various configurations of stimulation. The main characteristics of $R \& S T I M 12$ are the following:

○ Maximum output current: $\operatorname{Imax}=5 \mathrm{~mA}$,

○ Current resolution: $\Delta \mathrm{I}=1.3 \mu \mathrm{A}$

○ Temporal resolution: $\Delta \mathrm{t}=1 \mu \mathrm{s}$

○ Maximum pulse width: $T \max =2 \mathrm{~ms}$, 
Exploring selective neural electrical stimulation for upper limb function restoration

Eur J Transl Myol 26 (2) 161-164

Table 1. - Examples of stimulation configurations

\begin{tabular}{|l|l|l|l|l|l|l|l|l|l|l|l|l|}
\hline Name of the configuration - Number of Pole & 1 & 2 & 3 & 4 & 5 & 6 & 7 & 8 & 9 & 10 & 11 & 12 \\
\hline Ring Longitudinal & 2 & 1 & 2 & 1 & -3 & -3 & -3 & -3 & 2 & 1 & 2 & 1 \\
\hline Tripolaire TransverseA & 0 & 0 & 0 & 0 & 6 & -12 & 6 & 0 & 0 & 0 & 0 & 0 \\
\hline Tripolaire TransverseB & 6 & -12 & 6 & 0 & 0 & 0 & 0 & 0 & 0 & 0 & 0 & 0 \\
\hline Tripolaire TransverseC & 0 & 0 & 0 & 0 & 0 & 0 & 0 & 0 & 6 & -12 & 6 & 0 \\
\hline 1 Cathode Ring Anode & 1 & 2 & 1 & 2 & -12 & 0 & 0 & 0 & 6 & 0 & 0 & 0 \\
\hline Tripolaire Longitudinal & 6 & 0 & 0 & 0 & -12 & 0 & 0 & 0 & 6 & 0 & 0 & 0 \\
\hline Steering & 1 & 0 & 1 & 1 & 0 & -12 & 0 & 6 & 1 & 0 & 1 & 1 \\
\hline
\end{tabular}

Selective configuration design

After a simulation study, the more interesting combinations of poles from a selectivity performance point of view, were determined leading to up to 40 configurations to be tested on each nerve. When relevant, the same configurations of stimulation are reproduced on the 3 rings to investigate possible fascicular re-organization within the few millimeters which separate each ring (see Table 1).

Protocol and methodology

Cuff electrodes are placed around the median and/or radial nerves. Nerves are stimulated with increasing intensity. The protocol consists of the activation of one or more poles of the electrode. The stimulation pulse is biphasic, balanced but asymmetric, followed by a passive discharge to guaranty charge balance. Pulse width is fixed and intensity is modulated (up to $2.4 \mathrm{~mA}$ ). For each configuration and intensity, a tetanic stimulus is induced for 2 seconds. For the first patient pulse width was $500 \mu \mathrm{s}$ and stimulation frequency $25 \mathrm{~Hz}$. Cuff electrode did not fit perfectly, shorter pulse widths did not produced any movements. Surface EMG electrodes are placed upon the ERCL or ERCB, EDP, EDS, FDS, FDS and FPL muscles to record EMG signals. This allows us to evaluate the selectivity capacity of our configurations. Nevertheless, because the radial and median nerves activate more muscles than the recorded ones, in particular deep muscles contraction may not be detected, a synchronized video recording with the stimulator is performed. The video analysis is used to assess the functional selectivity capacity of our different stimulation configurations based on hand and wrist movements analysis

\section{Judgment criteria}

The primary judgment criterion is based on the quantification of the strength and the selectivity of muscle recruitment induced by the electrical stimulation of the median and/or radial nerves. The strength of recruitment and the selectivity are quantified for ECRL or ECRB, EDC, EPL, FPL, FDS and FDP muscles using electromyography.

For a given configuration $C$ and intensity $I$, a signal which reflects muscle activation is obtained for each muscle. Amplitudes of compound muscle action potentials (CMAP) are measured from the reference to the highest magnitude of the M-wave negative voltage. Then, for each configuration, those signals are normalized to the maximal amplitude of the CMAPs. We note the normalized signal $C M A P-E M G_{C I}$. CMAP$E M G_{C I}$ below 0.05 are considered equal to 0 . Intensities of stimulation corresponding to 20,50 and $100 \%$ of EMGcmax are determined for each configuration and for each of the 6 muscles. For each configuration, up to 18 series (6 muscles and 3 levels of activation) can be determined. For each series, an index of selectivity $\mathrm{SI}_{\mathrm{m}}(\mathrm{I}) \%$ is determined. $\mathrm{SI}_{\mathrm{m}}(\mathrm{I}) \%$ is defined as the ratiobetween the normalized signal $C M A P-E M G_{C I}$ of the $\mathrm{m}$ muscle whose nerve was stimulated with the optimal intensity $I$ causing an activation of $\mathrm{n}_{\%}\left(\mu_{\mathrm{i}}\right)$ and the sum of normalized signal $C M A P-E M G_{C I}\left(\mu_{\mathrm{j}}\right)$ of the 6 muscles:

$$
S I_{m}(I)_{\%}=\frac{\mu_{i}}{\sum_{j=1}^{6} \mu_{j}}
$$

$\mathrm{SI}_{\mathrm{m}}(\mathrm{I}) \%$ corresponds to the selectivity index. $\mathrm{SI}_{\mathrm{m}}(\mathrm{I}) \%$ is between 0 et 1 , where 0 indicates no selectivity and 1 indicates that only the muscle $m$ is activated. A selectivity index curve is plotted, from $\mathrm{SI}_{\mathrm{m}}(\mathrm{I}) \%$. The surgeon also evaluates muscle strength produced by the electrical stimulation (MRC scale). Finally, we have developed a software to track hand movements in the recorded video in order to qualify the motion range and type.

\section{Results}

We included 2 patients. Selective activation of the flexor carpi radialis and palmaris longus muscles was possible for the patient 1 from $600 \mu \mathrm{A}$ with grade 3 on the MRC scale. Preliminary quantitative results will be presented at the 2016 IFESS Conference to be held June $7-9,2016$ at La Grande Motte, Montpellier, France.

\section{Discussion}

Currently, there is no more commercial implanted stimulation neuroprosthesis allowing to restore hand movements. If the results of this study will be positive, it could lead us to design a new neuroprosthesis based 


\section{Exploring selective neural electrical stimulation for upper limb function restoration}

Eur J Transl Myol 26 (2) 161-164

on nerve stimulation for grasp movements in high tetraplegic subjects recovery. Even if the results can not be conclusive to how well a cuff electrode will work in chronic condition to selectively activate a high number of muscle groups because in chronic condition the electrode-nerve interface will have different properties due to impedance changes for example, we expect that our number of contact and modification of stimulation parameters will selectively activate the same muscle groups. Thus, a device using such technology would, in combination with tendon transfer surgery, be materially lighter than those which existed, would require less time for its implementation, and less power for its operation. An interface for piloting the stimulation is studied in parallel. $^{15}$

\section{Contributions}

WT: protocol design, experimental setup implementtation, data acquisition; DG: coordination of the technical and theoretical aspects of the study. Data acquisition and processing expertise; DA: technical expertise in stimulator design and programming; $\mathrm{BC}$ : neurosurgery and patient follow up; AG: patient recruitment, muscle mapping and patient follow up; $\mathrm{CF}$ : study design, expertise in FES applied to SCI rehabilitation; PM: stimulator design and stimulation pattern software programming; CP: experimental setup and data analysis support; OR: stimulation patterns definition and data processing; JT: neurosurgery and patient follow up; CA: coordination of the experimental aspects of the study. Protocol and setup design expertise.

\section{Acknowledgements}

The authors wish to thanks the subjects who participated in this study, S. Henkous, for her help in the writing of the protocol, as well as V. Leynaert, JL Divoux, MXMAxonic and ANRT support the PhD grant, CIFRE \#2013/0867.

\section{Conflict of Interest}

The authors declare no potential conflict of interests.

\section{Corresponding Author}

Wafa Tigra, INRIA, LIRMM, Montpellier, France.

E-mail: wafa.tigra@lirmm.fr

E-mails of Authors

David Guiraud: David.Guiraud@inria.fr

David Andreu: David.Andreu@lirmm.fr

Bertand Coulet: b-coulet@chu-montpellier.fr

Anthony Gélis: A.GELIS@ @ropara.fr

Charles Fattal: cfattal@cos-asso.org

Pawel Maciejasz: pmaciejasz@axonic.fr

Chloé Picq: cpicq@axonic.fr

Olivier Rossel: Olivier.Rossel@lirmm.fr

Jacques Teissier: jacques.teissier@wanadoo.fr

Christine Azevedo: Christine.Azevedo@inria.fr

\section{References}

1. Snoek GJ, IJzerman MJ, Hermens HJ, Maxwell D, Biering-Sorensen F. Survey of the needs of patients with spinal cord injury: impact and priority for improvement in hand function in tetraplegics," Spinal cord 2004;42:526-32.

2. Anderson KD. Targeting recovery: priorities of the spinal cord-injured population. J Neurotrauma 2004;21:1371-83.

3. Hanson R, Franklin M. Sexual loss in relation to other functional losses for spinal cord injured males. Arch Phys Med Rehabil 1976;57: 291.

4. Estores IM, The consumer's perspective and the professional literature: what do persons with spinal cord injury want? J Rehabil Res Dev 2003;40:93-8.

5. Keith MW, Peckham PH, Thrope GB, et al. Implantable functional neuromuscular stimulation in the tetraplegic hand. J Hand Surg Am 1989;14:524-30.

6. Billian C, Gorman PH. Upper extremity applications of functional neuromuscular stimulation. Assist Technol 1992;4:31-9.

7. Keith MW. Neuroprostheses for the upper extremity. Microsurgery 2001;21:256-63.

8. Hoshimiya N, Naito A, Yajima M, Handa Y. A multichannel FES system for the restoration of motor functions in high spinal cord injury patients: a respiration-controlled system for multijoint upper extremity. IEEE transactions on bio-medical engineering. 1989;36:754-60.

9. Peckham PH, Keith MW, Kilgore KL, et al. Efficacy of an implanted neuroprosthesis for restoring hand grasp in tetraplegia: a multicenter study Arch Phys Med Rehabil 2001;82:1380-8.

10. Hobby J, Taylor P, Esnouf J. Restoration of tetraplegic hand function by use of the neurocontrol freehand system. J Hand Surg $\mathrm{Br}$ 2001;26:459-64.

11. Lamb DW, Chan K. Surgical reconstruction of the upper limb in traumatic tetraplegia. J Bone Joint Surg 1983;65:291-8.

12. Mohammed KD, Rothwell AG, Sinclair SW, Willems S, Bean AR. Upper-limb surgery for tetraplegia. J Bone Joint Surg Br 1992;74:873-9.

13. Hovius S. Musculo-tendinous transfers of the hand and forearm. Clin Neurol Neurosurg 1993;95:S92-4. Review.

14. Tigra W, Azevedo Coste C, Fattal C, Guiraud D. Ergonomics of the control by a quadriplegic of hand functions, in Neural Engineering (NER), 7th Int. IEEE/EMBS Conf, 2015, 759-62. 